\title{
Capybara Oil Improves Hepatic Mitochondrial Dysfunction, Steatosis, and Inflammation in a Murine Model of Nonalcoholic Fatty Liver Disease
}

\author{
Polyana C. Marinho ${ }^{1 D},{ }^{1}$ Aline B. Vieira ${ }^{D},{ }^{2}$ Priscila G. Pereira, ${ }^{1}$ \\ Kíssila Rabelo, ${ }^{1}$ Bianca T. Ciambarella, ${ }^{1}$ Ana L. R. Nascimento, ${ }^{1}$ Erika Cortez $\left(\mathbb{D},{ }^{3}\right.$ \\ Aníbal S. Moura, ${ }^{4}$ Fernanda V. Guimarães, ${ }^{5}$ Marco A. Martins, ${ }^{5}$ \\ Gonzalo Barquero, ${ }^{6}$ Rodrigo N. Ferreira, ${ }^{7}$ and Jorge J. de Carvalho ${ }^{1}$ \\ ${ }^{1}$ Laboratory of Ultrastructure and Tissue Biology, Institute of Biology, State University of Rio de Janeiro, Rio de Janeiro, RJ, Brazil \\ ${ }^{2}$ Biomedical Sciences Department, Ross University School of Veterinary Medicine, Basseterre, Saint Kitts and Nevis \\ ${ }^{3}$ Laboratory of Stem Cells Research, Institute of Biology, State University of Rio de Janeiro, Rio de Janeiro, RJ, Brazil \\ ${ }^{4}$ Laboratory of Nutrition and Development Physiology, Institute of Biology, State University of Rio de Janeiro, Rio de Janeiro, RJ, Brazil \\ ${ }^{5}$ Laboratory of Inflammation, Oswaldo Cruz Institute, Fiocruz, Rio de Janeiro, RJ, Brazil \\ ${ }^{6}$ Tropical Sustainability Institute, São Paulo, SP, Brazil \\ ${ }^{7}$ College of Valença, Dom André Arcoverde Educational Foundation, Valença, RJ, Brazil
}

Correspondence should be addressed to Aline B. Vieira; avieira@rossvet.edu.kn

Received 24 January 2018; Revised 9 March 2018; Accepted 21 March 2018; Published 29 April 2018

Academic Editor: Yuan Xu

Copyright (C) 2018 Polyana C. Marinho et al. This is an open access article distributed under the Creative Commons Attribution License, which permits unrestricted use, distribution, and reproduction in any medium, provided the original work is properly cited.

Nonalcoholic fatty liver disease (NAFLD) is recognized as the most common cause of liver dysfunction worldwide and is commonly associated with obesity. Evidences suggest that NAFLD might be a mitochondrial disease, which contributes to the hepatic steatosis, oxidative stress, cytokine release, and cell death. Capybara oil (CO) is a rich source of polyunsaturated fatty acids (PUFA), which is known to improve inflammation and oxidative stress. In order to determine the effects of CO on NAFLD, C57Bl/6 mice were divided into 3 groups and fed a high-fat diet (HFD) (NAFLD group and NAFLD + CO group) or a control diet (CG group) during 16 weeks. The CO $(1.5 \mathrm{~g} / \mathrm{kg} /$ daily) was administered by gavage during the last 4 weeks of the diet protocol. We evaluated plasma liver enzymes, hepatic steatosis, and cytokine expression in liver as well as hepatocyte ultrastructural morphology and mitochondrial function. $\mathrm{CO}$ treatment suppressed hepatic steatosis, attenuated inflammatory response, and decreased plasma alanine aminotransferase (ALT) in mice with NAFLD. CO was also capable of restoring mitochondrial ultrastructure and function as well as balance superoxide dismutase and catalase levels. Our findings indicate that CO treatment has positive effects on NAFLD improving mitochondrial dysfunction, steatosis, acute inflammation, and oxidative stress.

\section{Introduction}

Nonalcoholic fatty liver disease (NAFLD) is the accumulation of fat in the liver in patients who do not consume excessive alcohol [1]. The term NAFLD encompasses a wide spectrum of conditions, from simple accumulation of fat ("fatty liver" or steatosis) to steatohepatitis, fibrosis, and cirrhosis with its clinical consequences [2]. NAFLD affects about $30 \%$ of the general population in western society and is recognized as the most common cause of liver dysfunction worldwide $[3,4]$.

NAFLD is asymptomatic in most affected patients and characterized as the presence of more than 5\% of lipid accumulation in the hepatocytes, excluding other liver disease etiologies (virus, autoimmune, alcohol, drugs, and genetics). NAFLD can progress to an advanced form, nonalcoholic steatohepatitis (NASH), which is defined by histological 
findings of hepatic steatosis, with hepatocyte damage and or inflammation [5-7]. Although uncommon, both NAFLD and NASH can further develop into liver fibrosis, cirrhosis, and eventually hepatocellular carcinoma $[8,9]$.

In addition to genetic risk factors, the prevalence of NAFLD is aggravated by changes in life style including physical inactivity as well as consumption of high fat and high fructose corn syrup diet [10]. Recently, NAFLD has been considered as a hepatic manifestation of metabolic syndrome (MS) which is defined by the presence of central obesity, insulin resistance, hyperlipidemia, hyperglycemia, and hypertension [3,11-13]. NAFLD induces insulin resistance and exacerbates systemic chronic inflammation and oxidative stress, which leads to organ dysfunction in extrahepatic tissues. There is now growing evidence that NAFLD is actually a multisystem disease, affecting several extrahepatic organs and regulatory pathways $[14,15]$.

Evidence suggests that NAFLD might be a mitochondrial disease. Mitochondrial dysfunction seems to play a role in the pathogenesis of NAFLD since it affects hepatic lipid homeostasis and promotes reactive oxygen species (ROS) production and lipid peroxidation, cytokine release, and cell death [16-18]. Mice with mitochondrial dysfunction develop NAFLD and hepatic insulin resistance independent of obesity or high-fat diet (HFD) [19, 20].

Although there is no specific effective treatment available for NAFLD, dietary therapy already showed beneficial results [21]. Administration of polyunsaturated fatty acids (PUFA) improves plasma lipid profile and may be useful in the treatment of NAFLD $[22,23]$. A recent systematic review and meta-analysis about the efficacy of PUFA in NAFLD derived from clinical trials confirmed an improvement in patients with this condition. The clinical improvement was based on hepatic steatosis and no other clinical or histological aspects were evaluated [24].

The oil derived from capybara (Hydrochoerus hydrochaeris) (CO), the largest rodent in the world, is a rich source of PUFA and contains $17.9 \%$ of alpha-linoleic and $19.6 \%$ linoleic fat acids (FAs), which correspond to essential omega-3 (n-3) and omega- 6 (n-6) FAs, respectively. In a previous study, CO reduced cholesterol blood levels in rats fed with cholesterolenriched diets [25]. The aim of this study was to determine the effect of $\mathrm{CO}$ on hepatic mitochondrial dysfunction, steatosis, and inflammation in a murine model of NAFLD.

\section{Materials and Methods}

2.1. Animals and Capybara Oil. This study was performed according to protocols (CEUA/015/2013) approved by the local Ethics Committee for Use and Care of Experimental Animals of the State University of Rio de Janeiro. All animals were maintained in $21 \pm 2^{\circ} \mathrm{C}, 60 \% \pm 10 \%$ of humidity, and $12: 1$ dark-light cycle. Capybara fat was donated by a private slaughter farm in Brazil that breed capybara in captivity as authorized by the Brazilian Institute of Environment and Renewable Natural Resources. The CO composition was previously described [25]. Oil was extracted using hydrothermal processing of the fat in a water bath [26].
2.2. NAFLD Induction and Capybara Oil Treatment. Thirty C57BL/6 male mice ( 8 weeks old) were divided into three groups ( $n=10$ per group): control group (CG), NAFLD group (NAFLD), and NAFLD treated with CO group (NAFLD + CO). CG animals were fed a control diet $(70 \%$ carbohydrates, $10 \%$ fat, and $20 \%$ proteins), and NAFLD animals were fed a HFD (29\% carbohydrates, 55\% fat, and $16 \%$ proteins) during 16 weeks in order to induce NAFLD [27]. The NAFLD + CO group received CO by oral gavage $(1.5 \mathrm{~g} / \mathrm{kg} /$ daily) during the last 4 weeks of the diet protocol while CG and NAFLD groups received water by gavage. One day after the last day of treatment, the animals were euthanized.

2.3. Sample Collection. After the last day of treatment, mice were fasted for 6 hours and anesthetized (xylazine $2 \mathrm{mg} / \mathrm{Kg}$ and ketamine $5 \mathrm{mg} / \mathrm{Kg}$ ). Blood samples were collected by cardiac puncture and transferred to heparinized tubes. Samples were then centrifuged $(1500 \mathrm{~g} / 10 \mathrm{~min})$ to obtain plasma which were stored at $-80^{\circ} \mathrm{C}$ until biochemical analyses. Liver samples were also collected and handled according to the specific techniques. Some samples were immediately used for high resolution respirometry while others were either frozen or fixed in different solutions for further analyses.

2.4. Serum Liver Enzymes. Plasma liver enzymes aspartate aminotransferase (AST) and alanine aminotransferase (ALT) were measured by a colorimetric enzymatic assay according to manufacturer's recommendations (Bioclin, Brazil).

2.5. Steatosis Quantification. Liver fragments fixed in paraformaldehyde $(4 \% \mathrm{w} / \mathrm{v})$ were embedded in paraffin, sectioned at a nominal thickness of five $\mu \mathrm{m}$, and stained with hematoxylin and eosin (HE). Ten digital images per animal were analyzed in a random and blinded manner and studied to assess steatosis as previously described [28]. Briefly, we used a 36-point test-system (PT) produced by the STEPanizer web-based software (http://www.stepanizer.com). The volume density $(\mathrm{Vv})$ of liver steatosis was estimated by pointcounting the fat droplets in hepatocytes: $\mathrm{Vv}$ [steatosis] $=\mathrm{PP}$ [steatosis]/PT (2) where PP is the number of points that defined steatosis $[28,29]$. This method was proved to be more consistent in assessing the hepatic steatosis than the use of scores [28].

2.6. Immunohistochemistry. Liver sections had antigen retrieval performed by citrate buffer at $\mathrm{pH} 6.0$ incubation for $30 \mathrm{~min}$ at $60^{\circ} \mathrm{C}$. Endogenous peroxidase activity was blocked using $0.3 \%$ hydrogen peroxide $\left(\mathrm{H}_{2} \mathrm{O}_{2}\right)$ and nonspecific binding of the polyclonal antibodies was blocked by incubation $5 \%(\mathrm{w} / \mathrm{v})$ bovine serum albumin (BSA) diluted in phosphate buffered saline (PBS). Subsequently, sections were incubated with antibodies, and these reactions were amplified using a biotin-streptavidin system (Dako, USA). Immunoreactive products were visualized using diaminobenzidine (DAB) reagent (Dako) and counterstained with hematoxylin. We used antitumor necrosis factor alpha (TNF- $\alpha$ ), and antitransforming growth factor beta (TGF- $\beta$ ) (dilution 1:100, Dako) antibodies. The estimated density per area $\left(Q_{A}\right)$ of TNF- $\alpha$ 
and TGF- $\beta$ positive cells was defined as cell numerical profile within a known test area (expressed as cells $/ \mu \mathrm{m}^{2}$ ) of $3305 \mu \mathrm{m}^{2}$ [26] using Image Pro Plus software. All analysis was held in blind test.

2.7. Ultrastructural Analyses. Liver samples were fixed in $2.5 \%$ glutaraldehyde (Sigma-Aldrich, Germany) in $0.1 \mathrm{~mol} / \mathrm{L}$ cacodylate buffer, post-fixed in $1 \%$ osmium tetroxide (SigmaAldrich), and embedded in Epon (Embed-812, USA). Ultrathin sections (60 to $70 \mathrm{~nm}$ ) were obtained from selected areas using an ultramicrotome (Leica, Austria), contrasted with uranyl acetate and lead citrate, and examined with a transmission electron microscope (TEM) (Jeol 1001, Japan) at $80 \mathrm{kV}$.

2.8. Oxidative Stress Analyses. Frozen liver homogenates were used for biochemical assays, as performed by KennedyFeitosa et al., 2016 [30]. Superoxide dismutase (SOD) activity was assayed by monitoring adrenaline (Sigma-Aldrich, USA) inhibition autooxidation. Catalase (CAT) activity was measured by a decrease of $\mathrm{H}_{2} \mathrm{O}_{2}$ (Sigma-Aldrich, USA) rate, and concentrations were monitored. As an index of oxidative damage induced by lipid peroxidation, we used thiobarbituric acid reactive substances (TBARS) (EMD Millipore, USA) method to analyze malondialdehyde (MDA) products during an acid-heating reaction. TBARS levels were expressed as MDA equivalents.

2.9. Mitochondrial Respirometry. We used high-resolution respirometry protocol according to Cortez et al., 2012 [31]. Briefly, liver fragments were mechanically macerated by using sharpened forceps and transferred into vessels with cooled Mitochondrial Respiration Medium, MIR05. Respiratory rates of liver were measured with Oroboros $2 \mathrm{k}$ Oxygraph (Oroboros Instruments, Austria) in $2 \mathrm{ml}$ of MIR05 at $37^{\circ} \mathrm{C}$ with continuous stirring. Matlab software (Oroboros Instruments) was used for data acquisition and analysis. Studies were performed with two independent sets of substrates, the carbohydrate $(\mathrm{CHO})$ and FA protocol. Respiratory parameters were defined as follows: maximally adenosine diphosphate- (ADP-) $(5 \mathrm{mM})$ stimulated respiration rates (State 3) and respiration rates in ADP phosphorylation absence and measured in presence of $1 \mu \mathrm{g} / \mathrm{ml}$ oligomycin (State 4). From respiratory fluxes obtained during substrate titration protocol, respiratory control ratio (RCR) was calculated for State 3/State 4.

2.10. Statistical Analyses. A statistical software (GraphPad Prism 6) was used for statistical analyses and graphics generation. Data is presented as means \pm standard error of the mean (SEM). Analysis of variance (one-way ANOVA) was performed followed by Bonferroni test. A $p$ value less than 0.05 was considered statistically significant.

\section{Results}

3.1. Serum Liver Enzymes. The levels of ALT (Table 1) were significantly increased $(p<p=0.026)$ in the NAFLD group $(25.0 \pm 6.4 \mathrm{mg} / \mathrm{dL})$ compared to the CG group $(6.8 \pm$
TABLE 1: Plasma liver enzymes of C57BL/6 mice treated with capybara oil.

\begin{tabular}{lccc}
\hline \multirow{2}{*}{ Parameters } & \multicolumn{3}{c}{ Groups } \\
& CG & NAFLD & NAFLD + CO \\
\hline ALT (mg/dl) & $6,8 \pm 0,9$ & $25,0 \pm 6,4^{\mathrm{a}}$ & $13,4 \pm 3,8^{\mathrm{b}}$ \\
AST (mg/dl) & $55,0 \pm 4,9$ & $59,0 \pm 5,9$ & $57,8 \pm 4,5$ \\
\hline
\end{tabular}

Alanine aminotransferase (ALT); aspartate aminotransferase (AST); control group (CG); NAFLD group (NAFLD); NAFLD treated with CO group $(\mathrm{NAFLD}+\mathrm{CO})$. Data were expressed as mean \pm SEM. Statistical analyses were performed using a one-way ANOVA analysis of variance followed by Bonferroni test. a represents $p=0.026$ compared to CG group and $\mathrm{b}$ represents $p=0.046$ compared to NAFLD group.

$0.9 \mathrm{mg} / \mathrm{dL}$ ). Four weeks of treatment with CO reduced ALT levels in $46.4 \%(13.4 \pm 3.8)$. No differences were found in the levels of AST (Table 1) between groups (CG $=55.0 \pm 4.9$; $\mathrm{NAFLD}=59.0 \pm 5.9 ; \mathrm{NAFLD}+\mathrm{CO}=57.8 \pm 4.5)$.

3.2. Steatosis Quantification. Animals from CG showed a low number of fat droplets (Figure 1(a)) and a small steatosis percentage $(\mathrm{CG}=2.94 \pm 1.35 \%$ ) (Figure 1(b)). Animals fed a high fat diet without treatment showed severe macroand microlipid droplets in hepatocytes (Figure 1(a)) and significantly increasing steatosis density $(\mathrm{NAFLD}=18.89$ $\pm 2.32 \%$ ) (Figure 1(b)). Treatment with CO decreased the severity of lipid droplets in hepatocytes (Figure 1(a)) and consequently the percentage of fat accumulation (NAFLD + $\mathrm{CO}=4.97 \pm 1.92 \%)$ (Figure 1(b)).

3.3. Immunohistochemistry. Immunohistochemistry staining was performed in liver tissue to analyze the effect of CO on the inflammatory response induced by NAFLD. TNF$\alpha$ expression significantly increased in NAFLD group (6.93 \pm 0.75 positive cells/fields) compared to CG $(1.53 \pm 0.44$ positive cells/fields) (Figure 1(a)). Likewise, the expression of TGF- $\beta$ increased significantly in NAFLD group $(5.50 \pm 0.62$ positive cells/fields) compared to CG $(0.83 \pm 0.47$ positive cells/fields) (Figure 1(a)). Treatment with CO significantly decreased the TNF- $\alpha$ expression (NAFLD $+\mathrm{CO}=3.87 \pm 0.70$ positive cells/fields) (Figure 1(c)) but greatly increased TGF- $\beta$ expression (NAFLD $+\mathrm{CO}=14.17 \pm 0.83$ positive cells/fields) (Figure 1(d)).

3.4. Ultrastructural Liver Analyses. The electron microscopy analyses of CG showed preserved organelles and numerous well developed and organized mitochondria with an intact membrane and matrix (Figure 2(a)). The NAFLD group presented a decrease in mitochondrial matrix electron density and loss of membrane integrity. This membrane disruption leads to consequent mitochondrial matrix extravasation in the cytoplasm. Additionally, NAFLD group showed a remarkable disorganization in the cytoarchitecture and higher accumulation of glycogen in the cytoplasm (Figure 2(b)). On the other hand, hepatocytes of animals treated with $\mathrm{CO}$ exhibited numerous mitochondria and recovery of the mitochondrial membrane integrity and matrix electron density. Hepatic cells from NAFLD + CO group remained with organized 
HE
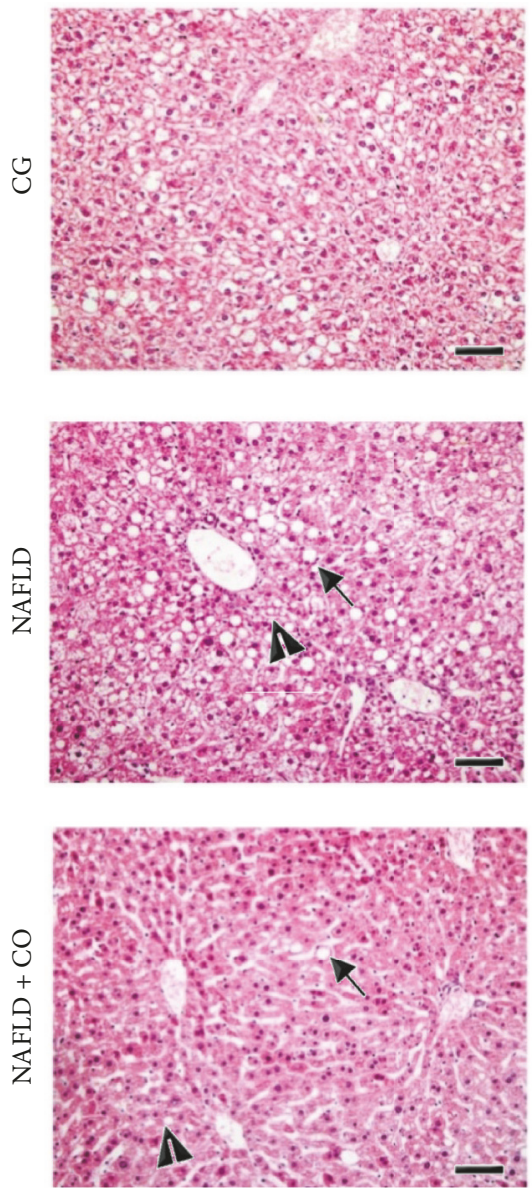

TNF- $\alpha$
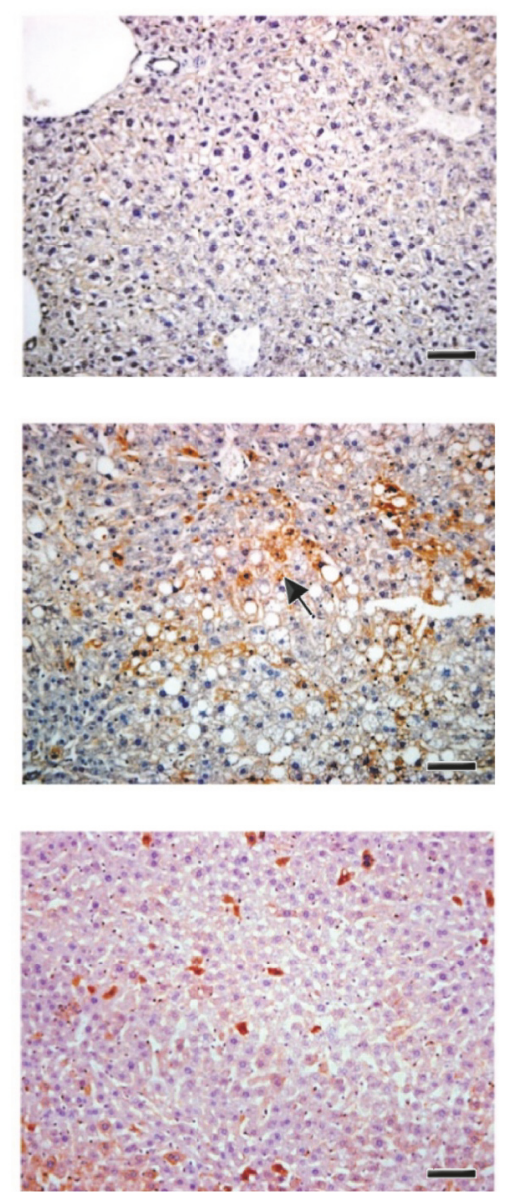

(a)

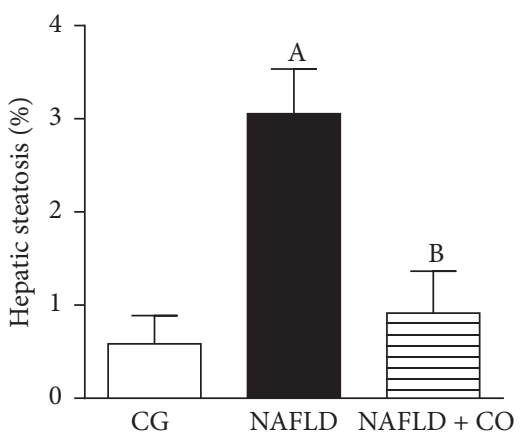

(b)

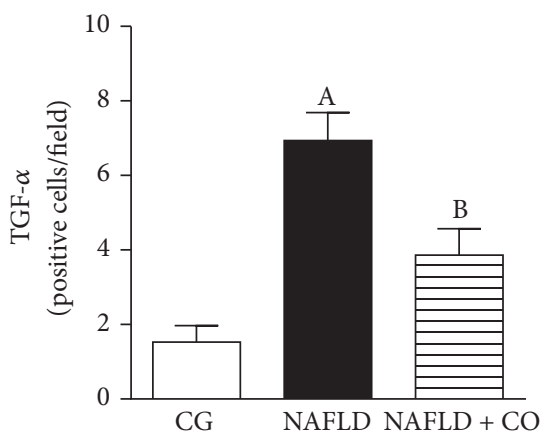

(c)
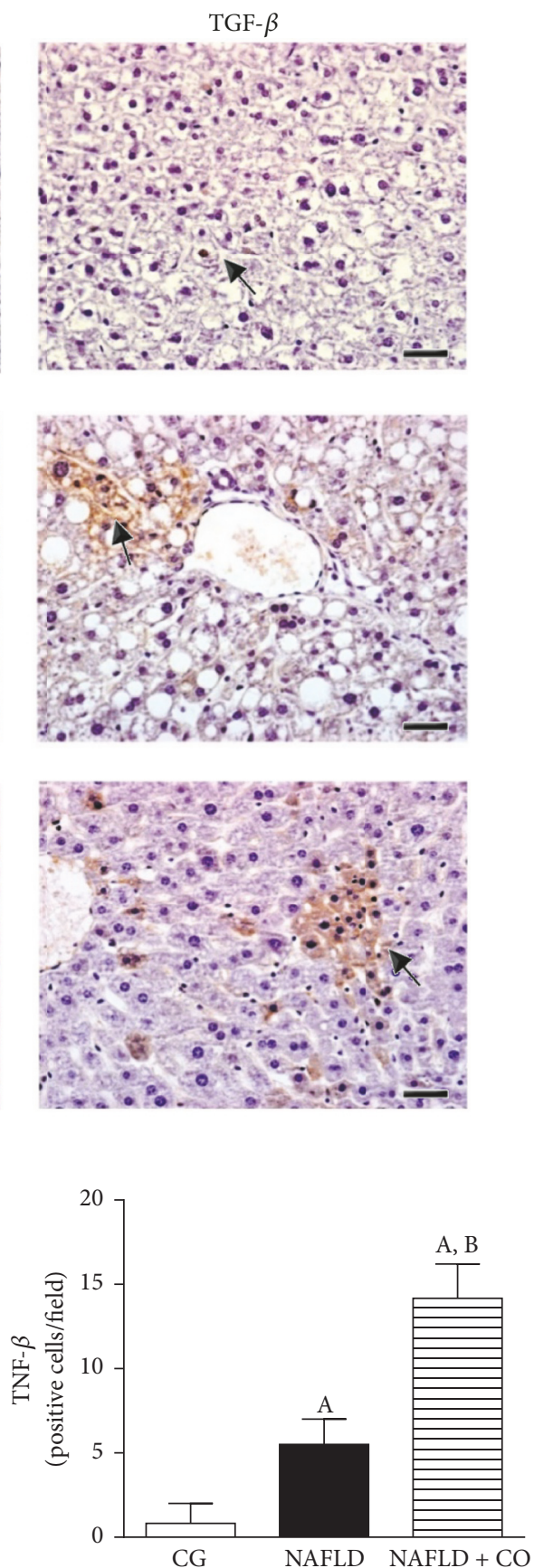

(d)

FIGURE 1: Morphological changes in the liver of C57BL/6 mice treated with capybara oil. (a, b, c, and d) (a) Panel with a photomicrograph of liver showing hepatic steatosis (HE), tumor necrosis factor-alpha (TNF- $\alpha$ ) and transforming growth factor-beta (TGF- $\beta$ ) expression. The black arrows indicate the macrovesicles and arrows head indicate the microvesicles (HE); the black arrows indicate immunostaining of TNF- $\alpha$ and immunostaining of TGF- $\beta$, respectively. Bar indicates $400 \mu \mathrm{m}$ to HE and TNF- $\alpha$ images and $200 \mu \mathrm{m}$ to TGF- $\beta$ images. (b) Evaluation of hepatic steatosis by morphometry. (c) Quantification of TNF- $\alpha$ by Immunohistochemistry. (d) Quantification of TGF- $\beta$ by Immunohistochemistry. Data are expressed as the mean \pm SEM. Statistical analyses were performed using a one-way ANOVA analysis of variance followed by Bonferroni test. (b) ${ }^{\mathrm{A}} p=0.0006$ compared to the CG group and ${ }^{\mathrm{B}} p=0.0021$ compared to NAFLD group. (c) ${ }^{\mathrm{A}} p<0.0001$ compared to the CG group and ${ }^{\mathrm{B}} p=0.0042$ compared to NAFLD group. (d) ${ }^{\mathrm{A}} p=0.0002$ compared to the CG group (in the NAFLD bar); ${ }^{\mathrm{A}} p<0.0001$ compared to the CG group (in NAFLD + CO bar) and ${ }^{\mathrm{B}} p<0.0001$ compared to NAFLD group. 


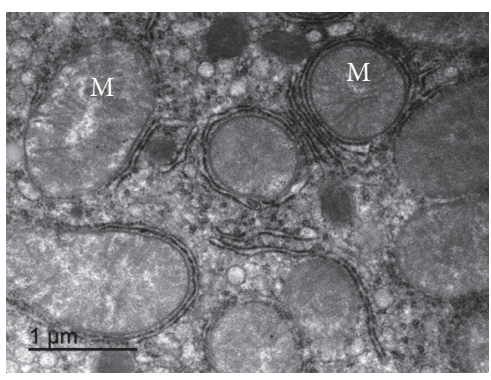

(a)

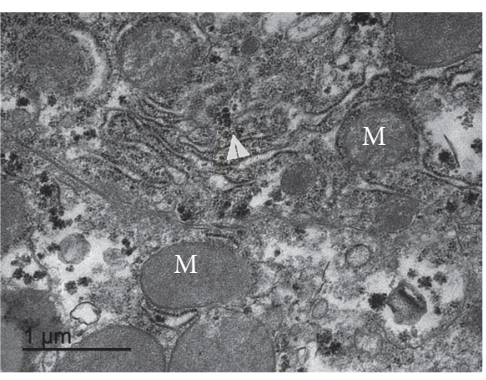

(b)

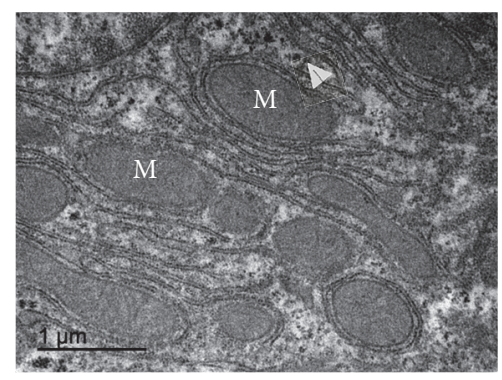

(c)

Figure 2: Mitochondrial morphological evaluation in the liver of C57BL/6 mice treated with capybara oil. (a, b, and c) Panel with ultraphotomicrograph of hepatocytes showing the mitochondrial morphology of CG, NAFLD, and NAFLD + CO, respectively; $M=$ mitochondria. Whitehead arrows indicate glycogen. Bars indicate $1 \mu \mathrm{m}$

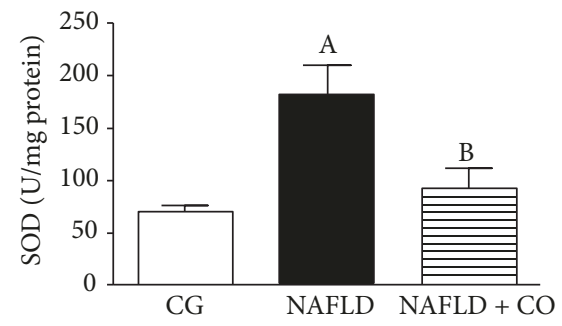

(a)

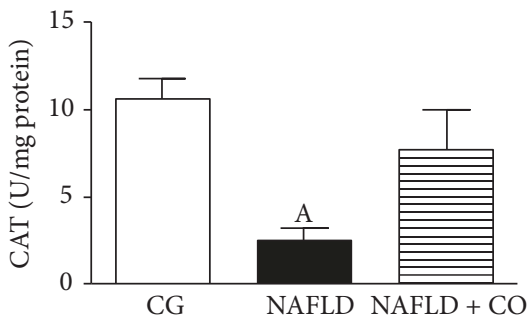

(b)

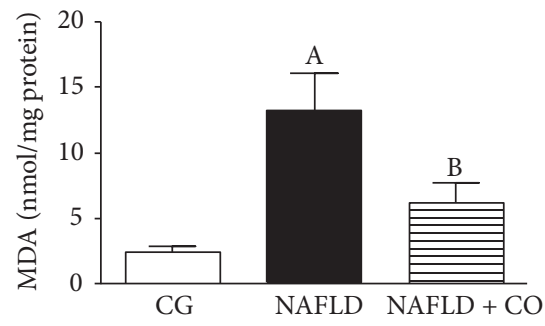

(c)

FIGURE 3: Oxidative stress evaluation in the liver of C57BL/6 mice treated with capybara oil. (a, b, and c) (a-c) represents antioxidants enzymes and lipid peroxidation products in serum, (a) superoxide dismutase (SOD) activity, (b) catalase (CAT) activity, and (c) malondialdehyde (MDA) levels. Data are expressed as mean \pm SEM. Statistical analyses were performed using a one-way ANOVA analysis of variance followed by Bonferroni test. (a) ${ }^{\mathrm{A}} p=0.0084$ compared to CG group; ${ }^{\mathrm{B}} p=0.0364$ compared to NAFLD group. (b) ${ }^{\mathrm{A}} p=0.0035$ compared to CG group. (c) ${ }^{\mathrm{A}} p=0.0006$ compared to CG group; ${ }^{\mathrm{B}} p=0.0264$ compared to NAFLD group.

ultrastructural arrangement compared to NAFLD group and showed less glycogen accumulation (Figure 2(c)).

3.5. Oxidative Stress Analysis. NAFLD group showed significantly increased SOD antioxidant enzyme activity (180.9 $\pm 27.46 \mathrm{U} / \mathrm{mg}$ protein) compared to the CG group (70.09 $\pm 5.35 \mathrm{U} / \mathrm{mg}$ protein). Treatment with $\mathrm{CO}$ significantly decreased SOD activity (NAFLD $+\mathrm{CO}=91.56 \pm 19.42 \mathrm{U} / \mathrm{mg}$ protein) compared with NAFLD group (Figure 3(a)). NAFLD group showed depressed CAT antioxidant enzymes activity $(2.53 \pm 0.63 \mathrm{U} / \mathrm{mg}$ protein) compared to CG group (10.6 \pm $1.14 \mathrm{U} / \mathrm{mg}$ protein). No significant difference was found in CAT activity after CO treatment (NAFLD + CO $=7.66 \pm$ $2.32 \mathrm{U} / \mathrm{mg}$ protein) compared to NAFLD group (Figure $3(\mathrm{~b})$ ). NAFLD group showed significantly increased in the MDA levels $(13.16 \pm 2.83 \mathrm{nmol} / \mathrm{mg}$ protein) compared to the CG group $(2.41 \pm 0.48 \mathrm{nmol} / \mathrm{mg}$ protein $)$. Treatment with $\mathrm{CO}$ significantly decreased MDA levels (NAFLD $+\mathrm{CO}=6.22 \pm$ $1.53 \mathrm{nmol} / \mathrm{mg}$ protein) compared with NAFLD group (Figure $3(\mathrm{c}))$.

3.6. Mitochondrial Respirometry. To evaluate mitochondrial function, respirometry experiments were performed. NAFLD animals without treatment showed liver mitochondrial impairment, with a reduction in maximum ADPstimulate respiratory rates (State 3 ) in oxidation protocols, $\mathrm{CHO}$, and FAs reduction, compared with CG. CO treatment
$(\mathrm{NAFLD}+\mathrm{CO})$ promoted a significant increase in State 3 respiration in $\mathrm{CHO}$ and FAs, respectively (Figures 4(a) and 4(b)). Furthermore, RCR was significantly decreased in $\mathrm{CHO}$ and FAs oxidation in NAFLD group compared to CG. After $\mathrm{CO}$ treatment an increase in RCR levels was observed similar to $\mathrm{CHO}$ oxidation in CG (Figure $4(\mathrm{c})$ ). The CO effect was not verified in relation to FAs oxidation (Figure 4(d)).

\section{Discussion}

The present study showed that $\mathrm{CO}$, an alternative PUFA source, was capable of reversing liver damage associated with NAFLD in obese mice. The oil was able to reduce plasmatic levels of ALT enzyme, decrease hepatic steatosis, and improve tissue morphology and inflammation. Moreover, CO improved abnormalities in mitochondrial ultrastructure, cellular respiratory efficiency, and imbalance of antioxidant enzymes.

Prolonged HFD in C57Bl/6 mice is an established model of diet-induced obesity [32]. Previous studies have demonstrated that HFD also leads to NAFLD $[33,34]$ and in humans can sometimes evolve to NASH [35]. As expected, animals submitted to the present HFD successfully developed features of NAFLD including liver steatosis, increased plasma liver enzymes, and inflammation compared with control animals. Although this model of NAFLD mimics both the histopathology and pathogenesis of human NAFLD [36] animals do not 


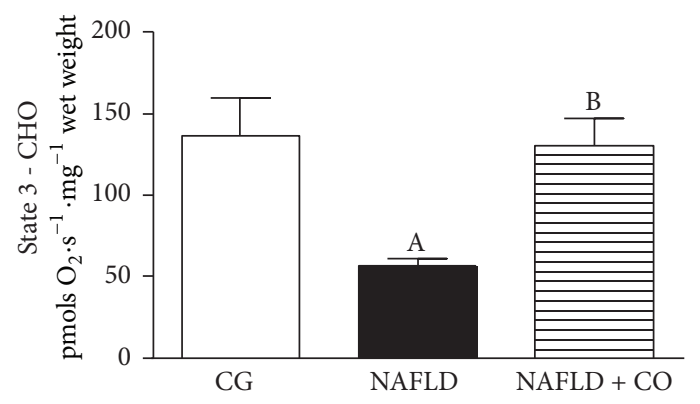

(a)

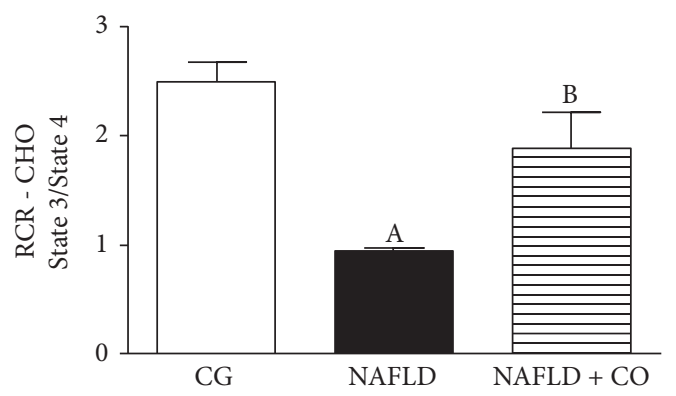

(c)

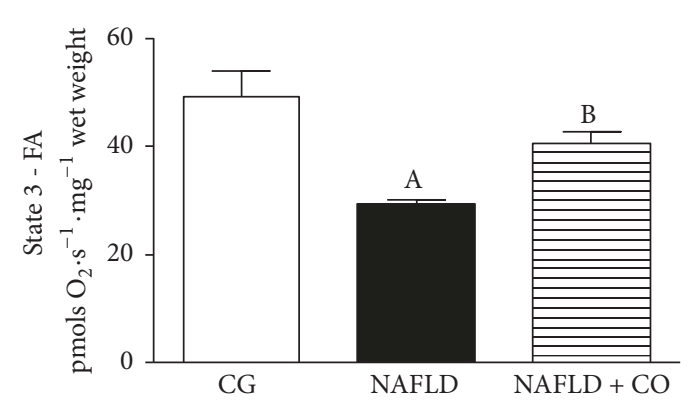

(b)

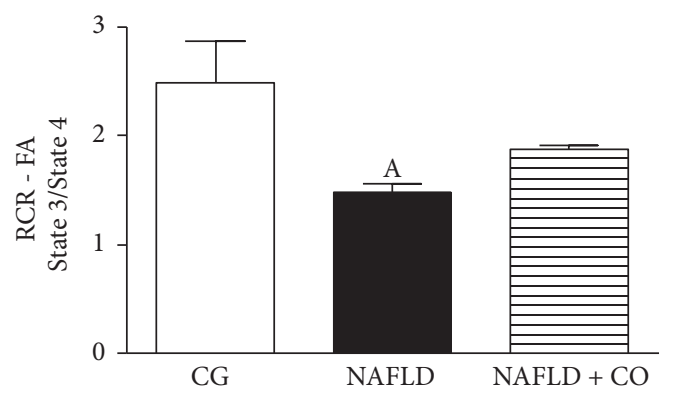

(d)

FIGURE 4: Mitochondrial functional evaluation in the liver of C57BL/6 mice treated with capybara oil. (a, b, c, and d) (a) represents highresolution respirometry in liver cells. (b) Maximal ADP-stimulated respiratory rates (State 3) with substrates related to carbohydrate oxidation, (c) maximal ADP-stimulated respiratory rates (State 3) with substrates related to fatty acid oxidation, (d) respiratory control ratio (RCR), and calculated as State 3/State 4 ratios for $\mathrm{CHO}$ and FAs oxidation, respectively. Data are expressed as mean \pm SEM. Statistical analyses were performed using a one-way ANOVA analysis of variance followed by Bonferroni test. (a) ${ }^{\mathrm{A}} p=0.0212$ compared to CG group; ${ }^{\mathrm{B}} p=0.0286$ compared to NAFLD group. (b) ${ }^{\mathrm{A}} p=0.0019$ compared to CG group; ${ }^{\mathrm{B}} p=0.0472$ compared to NAFLD group. (c) ${ }^{\mathrm{A}} p=0.0005$ compared to CG group; ${ }^{\mathrm{B}} p=0.0187$ compared to NAFLD group. (d) ${ }^{\mathrm{A}} p=0.0225$ compared to CG group.

develop NASH or fibrosis. Further investigation is necessary to determine if $\mathrm{CO}$ has any beneficial effects on progressive forms of NAFLD that may lead to fibrosis, cirrhosis, and ultimately hepatocellular carcinoma.

Aminotransferase levels have been used as a marker of NAFLD [37]. NAFLD is the commonest cause of elevated ALT and presumed liver injury $[38,39]$. ALT and/or AST levels seem to be predictive of the presence of NAFLD when other chronic liver diseases are excluded and features of MS like obesity are present [38]. There are two soluble ALT isoenzymes in mammals, cytosolic and mitochondrial, playing a role in amino acid metabolism and gluconeogenesis [40]. CO treatment successfully reduced plasmatic levels of ALT suggesting a recovery of enzyme leakage from damaged hepatocytes.

Fat accumulation in the liver of patients with NAFLD is mainly in the form of triglycerides [41, 42]. Triglyceride accumulation is not hepatotoxic per se and could represent a defensive mechanism to balance free fat acid (FFA) excess $[43,44]$. Previous evidences suggest that liver steatosis is an epiphenomenon, which happens simultaneously with toxic metabolites generation, lipotoxicity, and liver injury. Our data shows that $\mathrm{CO}$ treatment reduced liver steatosis in $73.69 \%$, which agrees with previous studies using PUFA in the treatment of NAFLD [22-24].

NAFLD is associated with the production and release of proinflammatory cytokines, both systemically and locally in the liver, such as TNF- $\alpha$ [45]. The persistence of this cytokine could lead to chronic inflammation with fibrosis development, as characterized in NASH condition [46]. Serum and hepatic levels of TNF- $\alpha$ are increased in patients with NASH and correlate with histological severity of liver damage [47]. We found hepatic levels of TNF- $\alpha$ to be increased in our model and CO significantly reduced hepatic TNF- $\alpha$ levels. The absence of NASH and fibrosis in our model is a limitation of this study. Nevertheless, this cytokine is known to contribute to mitochondrial dysfunction, inducing mitochondrial swelling, rarefaction of the matrix, and membrane burst interfering in mitochondrial respiratory complexes [48]. We also investigated the hepatic levels of TGF- $\beta$, a pleiotropic cytokine that plays a dual role in immune functions [49]. Biological actions of TGF- $\beta$ include regulation of cell proliferation, control of extracellular matrix protein production and degradation, cellular differentiation modulation, and either stimulatory or inhibitory effects on the same cell, depending on the cellular environment [50]. It is well stablished that n-3 PUFA is able to interact with peroxisome proliferator-activated receptor $\alpha(\operatorname{PPAR} \alpha)$ and inhibit factor nuclear kappa B (NF- $\kappa \mathrm{B})$ [51], resulting in reduction of TNF- $\alpha$ and interleukin (IL-6) expression in hepatocytes [52]. In adaptive immune response, the differentiation of (cluster of differentiation) $\mathrm{CD}^{+} \mathrm{T}$ cells subtypes in Th17 proinflammatory $\mathrm{T}$ cells is dependent of association between IL- 6 and TGF- $\beta$, while the anti-inflammatory 
Treg cells only need high levels of TGF- $\beta$ stimulation to differentiate [53]. This mechanism could be related to our data, suggesting that $\mathrm{CO}$ treatment, rich in n-3 PUFA, helped to decrease inflammation either through inhibition of NF- $\kappa \mathrm{B}$ (indirectly indicated by the decrease in TNF- $\alpha$ production) or by increasing TGF- $\beta$ levels in the liver (and probably the anti-inflammatory response stimulating Treg cells differentiation). However, more analyses are necessary to confirm this hypothesis.

Structural and functional alterations in mitochondria have showed to contribution in the pathogenesis of NAFLD. Structural alterations encompass depletion of mitochondrial deoxyribonucleic acid (DNA) and morphological and ultrastructural changes, while functional alterations include the respiratory chain and mitochondrial $\beta$-oxidation [52]. The mitochondrial respiratory efficiency loss seems to occur due to a possible uncoupling effect exerted by the excess of FAs present in the HFD $[54,55]$. We found several structural and functional derangements in our animals with NAFLD that were efficiently treated by CO. Whether mitochondrial dysfunction is a key pathogenic event in NAFLD or the consequence of an altered lipid metabolism remains unclear.

Oxidative stress results from an imbalance between ROS, prooxidant, and antioxidant chemical species that leads to cellular oxidative damage. The FAs oxidation is an important source of ROS in fatty livers [56] and oxidative stress exacerbation in NASH promotes hepatocellular damage by inducing severe alterations in biomolecules, with functions loss and cell viability [57]. The mitochondrial respiration dysfunction, observed in our study, can directly lead to ROS production. If electron flow is interrupted; respiratory intermediates transfer electrons to molecular oxygen producing superoxide anions and $\mathrm{H}_{2} \mathrm{O}_{2}$. With impaired mitochondrial oxidative capacity, cytosolic FAs accumulate [48]. The decrease of antioxidant CAT enzyme activities, found in the NAFLD group, seems to occur because of rapid consumption and exhaustion of these enzymes storage, a consequence of their role in avoiding free radicals generated in NAFLD. In our study, SOD enzyme activity was higher in NAFLD group in agreement with previous report using high fat diet [58]. High concentrations of antioxidant enzymes represent stress oxidation that the body tries to eliminate [59]. NAFLD increased liver oxidative stress as evidenced by the increase liver's oxidative damage (such as the increase in MDA level) and the inhibition of liver's antioxidant enzymes (CAT) [58]. Treatment with CO was able to significantly reverse MDA levels, consequently lipid peroxidation, and oxidative stress.

Recently, there was an increasing interest in the use of the PUFAs as a nutritional supplementation, since many studies have been proving protective effects in patients with different diseases including the ones related to obesity [60, 61]. Although some authors have found that PUFA treatment seems to be not effective in NASH $[62,63]$, its effects in early stages of the disease is undeniable reversing the hallmark features observed in human NAFLD patients. Whether or not the prevention of NALFD progression to NASH is relevant when treating obese patients is something that requires further investigation.
In most of the studies, PUFA sources are fish or plants where the extraction procedures are complex and result in low yields of the product $[59,60,64,65]$. We decided to investigate the CO, due to its high levels of PUFA and potential for becoming an alternative source of these FAs [25]. This oil has potential to be used for consumption in the food industry, rather than being discarded, as capybara meat is already marketed for consumption. Additional to the previous study showing beneficial effects on dyslipidemia [25], our group has also described that $\mathrm{CO}$ acts beneficially in cutaneous wound healing [26].

\section{Conclusion}

We conclude that CO treatment has positive effects on the benign form of obesity-induced NAFLD improving mitochondrial dysfunction, steatosis, acute inflammation, and oxidative stress in mice.

\section{Data Availability}

The data used to support the findings of this study will be available upon request. Please contact Dr. Jorge José de Carvalho, Head of the Laboratory of Ultrastructure and Tissue Biology at carvalho@uerj.edu.br.

\section{Conflicts of Interest}

The authors have no conflicts of interest to disclose.

\section{Acknowledgments}

This work was supported by the Fundação Carlos Chagas Filho de Amparo à Pesquisa do Estado do Rio de Janeiro (FAPERJ), Conselho Nacional de Desenvolvimento Científico e Tecnológico (CNPq), Coordenação de Aperfeiçoamento de Pessoal de nível Superior (Capes), Universidade do Estado do Rio de Janeiro (UERJ), and Ross University School of Veterinary Medicine.

\section{References}

[1] P. Angulo, "Medical progress: nonalcoholic fatty liver disease," The New England Journal of Medicine, vol. 346, no. 16, pp. 12211231, 2002.

[2] V. Ratziua, S. Bellentanib, and H. Cortez-Pintoc, "A position statement on NAFLD/NASH based on the EASL 2009 special conference," Journal of Hepatology, vol. 53, no. 2, pp. 372-384, 2010.

[3] N. Chalasani, Z. Younossi, and J. E. Lavine, "The diagnosis and management of non-alcoholic fatty liver disease: practice guideline by the American Association for the Study of Liver Diseases, American College of Gastroenterology, and the American Gastroenterological Association," Hepatology, vol. 55, no. 6, pp. 2005-2023, 2012.

[4] H. C. Masuoka and N. Chalasani, "Nonalcoholic fatty liver disease: an emerging threat to obese and diabetic individuals," Annals of the New York Academy of Sciences, vol. 1281, no. 1, pp. 106-122, 2013. 
[5] E. Tsochatzis, G. V. Papatheodoridis, E. K. Manesis, G. Kafiri, D. G. Tiniakos, and A. J. Archimandritis, "Metabolic syndrome is associated with severe fibrosis in chronic viral hepatitis and non-alcoholic steatohepatitis," Alimentary Pharmacology \& Therapeutics, vol. 27, no. 1, pp. 80-89, 2008.

[6] K. Hassan, V. Bhalla, M. E. El Regal, and H. H. A-Kader, "Nonalcoholic fatty liver disease: a comprehensive review of a growing epidemic," World Journal of Gastroenterology, vol. 20, no. 34, pp. 12082-12101, 2014.

[7] Z. M. Younossi, A. B. Koenig, D. Abdelatif, Y. Fazel, L. Henry, and M. Wymer, "Global epidemiology of nonalcoholic fatty liver disease-meta-analytic assessment of prevalence, incidence, and outcomes," Hepatology, 2015.

[8] R. Pais, L. Fartoux, C. Goumard et al., "Temporal trends, clinical patterns and outcomes of NAFLD-related HCC in patients undergoing liver resection over a 20-year period," Alimentary Pharmacology \& Therapeutics, vol. 46, no. 9, pp. 856-863, 2017.

[9] D. Goldberg, I. C. Ditah, K. Saeian et al., "Changes in the Prevalence of Hepatitis C Virus Infection, Nonalcoholic Steatohepatitis, and Alcoholic Liver Disease Among Patients With Cirrhosis or Liver Failure on the Waitlist for Liver Transplantation," Gastroenterology, vol. 152, no. 5, pp. 1090-1099.el, 2017.

[10] L. H. Tetri, M. Basaranoglu, E. M. Brunt, L. M. Yerian, and B. A. Neuschwander-Tetri, "Severe NAFLD with hepatic necroinflammatory changes in mice fed trans fats and a highfructose corn syrup equivalent," American Journal of PhysiologyGastrointestinal and Liver Physiology, vol. 295, no. 5, pp. G987G995, 2008.

[11] A. Alisi, M. Manco, N. Panera, and V. Nobili, "Association between type two diabetes and non-alcoholic fatty liver disease in youth," Annals of Hepatology, vol. 8, no. 1, pp. S44-S50, 2009.

[12] R. Parikh and M. V. Mohan, "Changing definitions of metabolic syndrome," Indian Journal of Endocrinology and Metabolism, vol. 16, no. 1, pp. 7-12, 2012.

[13] R. Rahman, G. M. Hammoud, A. A. Almashhrawi, K. T. Ahmed, and J. A. Ibdah, "Primary hepatocellular carcinoma and metabolic syndrome: An update," World Journal of Gastrointestinal Oncology, vol. 5, no. 9, pp. 186-194, 2013.

[14] M. J. Armstrong, L. A. Adams, A. Canbay, and W.-K. Syn, "Extrahepatic complications of nonalcoholic fatty liver disease," Hepatology, vol. 59, no. 3, pp. 1174-1197, 2014.

[15] E. Han and Y.-H. Lee, "Non-alcoholic fatty liver disease: The emerging burden in cardiometabolic and renal diseases," Diabetes \& Metabolism, vol. 41, no. 6, pp. 430-437, 2017.

[16] M. Lammens and H. Laak, "Contribution of histopathological examination to the diagnosis of OXPHOS disorders," in Oxidative Phosphorylation in Health and Disease, A. M. Smeitink, S. A. Sengers, and J. M. Trijbels, Eds., pp. 53-78, 2005.

[17] K. Begriche, A. Igoudjil, D. Pessayre, and B. Fromenty, "Mitochondrial dysfunction in NASH: causes, consequences and possible means to prevent it," Mitochondrion, vol. 6, no. 1, pp. $1-28,2006$.

[18] A. P. Rolo, J. S. Teodoro, and C. M. Palmeira, "Role of oxidative stress in the pathogenesis of nonalcoholic steatohepatitis," Free Radical Biology \& Medicine, vol. 52, no. 1, pp. 59-69, 2012.

[19] J. A. Ibdah, P. Perlegas, Y. Zhao et al., "Mice heterozygous for a defect in mitochondrial trifunctional protein develop hepatic steatosis and insulin resistance," Gastroenterology, vol. 128, no. 5, pp. 1381-1390, 2005.

[20] R. S. Rector, E. M. Morris, S. Ridenhour et al., "Selective hepatic insulin resistance in a murine model heterozygous for a mitochondrial trifunctional protein defect," Hepatology, vol. 57, no. 6, pp. 2213-2223, 2013.

[21] M. Del Ben, L. Polimeni, F. Baratta, D. Pastori, L. Loffredo, and F. Angelico, "Modern approach to the clinical management of non-alcoholic fatty liver disease," World Journal of Gastroenterology, no. 26, pp. 8341-8350, 2014.

[22] E. M. McCarthy and M. E. Rinella, "The Role of Diet and Nutrient Composition in Nonalcoholic Fatty Liver Disease," Journal of the Academy of Nutrition and Dietetics, vol. 112, no. 3, pp. 401-409, 2012.

[23] G. S. Masterton, J. N. Plevris, and P. C. Hayes, "Review article: omega-3 fatty acids-a promising novel therapy for non-alcoholic fatty liver disease," Alimentary Pharmacology \& Therapeutics, vol. 31, no. 7, pp. 679-692, 2010.

[24] H. M. Parker, N. A. Johnson, C. A. Burdon, J. S. Cohn, H. T. O'Connor, and J. George, "Omega-3 supplementation and non-alcoholic fatty liver disease: a systematic review and metaanalysis," Journal of Hepatology, vol. 56, no. 4, pp. 944-951, 2012.

[25] M. Fukushima, Y. Takayama, T. Habaguchi, and M. Nakano, "Comparative hypocholesterolemic effects of capybara (Hydrochoerus hydrochaeris dabbenei) oil, horse oil, and sardine oil in cholesterol-fed rats," Lipids, vol. 32, no. 4, pp. 391-395, 1997.

[26] P. C. Marinho, R. Neto-Ferreira, and J. José De Carvalho, "Evaluation of therapeutic intervention with a natural product in Cutaneous wound healing: The use of capybara oil," EvidenceBased Complementary and Alternative Medicine, vol. 2013, Article ID 217198, 10 pages, 2013.

[27] M. C. Calixto, L. Lintomen, A. Schenka, M. J. Saad, A. Zanesco, and E. Antunes, "Obesity enhances eosinophilic inflammation in a murine model of allergic asthma," British Journal of Pharmacology, vol. 159, no. 3, pp. 617-625, 2010.

[28] M. Catta-Preta, L. S. Mendonca, J. Fraulob-Aquino, M. B. Aguila, and C. A. Mandarim-De-Lacerda, "A critical analysis of three quantitative methods of assessment of hepatic steatosis in liver biopsies," Virchows Archiv, vol. 459, no. 5, pp. 477-485, 2011.

[29] F. A. M. Nascimento, S. Barbosa-da-Silva, C. Fernandes-Santos, C. A. Mandarim-de-Lacerda, and M. B. Aguila, "Adipose tissue, liver and pancreas structural alterations in C57BL/6 mice fed high-fat-high-sucrose diet supplemented with fish oil (n-3 fatty acid rich oil)," Experimental and Toxicologic Pathology, vol. 62, no. 1, pp. 17-25, 2010.

[30] E. Kennedy-Feitosa, R. T. Okuro, V. Pinho Ribeiro et al., "Eucalyptol attenuates cigarette smoke-induced acute lung inflammation and oxidative stress in the mouse," Pulmonary Pharmacology and Therapeutics, vol. 41, pp. 11-18, 2016.

[31] E. Cortez, F. A. Neves, A. F. Bernardo et al., "Lymphocytes mitochondrial physiology as biomarker of energy metabolism during fasted and fed conditions," The Scientific World Journal, vol. 2012, Article ID 629326, 2012.

[32] N. Hariri and L. Thibault, "High-fat diet-induced obesity in animal models," Nutrition Research Reviews, vol. 23, no. 2, pp. 270-299, 2010.

[33] J. C. Fraulob, R. Ogg-Diamantino, C. Fernandes-Santos, M. B. Aguila, and C. A. Mandarim-de-Lacerda, "A mouse model of metabolic syndrome: insulin resistance, fatty liver and NonAlcoholic Fatty Pancreas Disease (NAFPD) in C57BL/6 mice fed a high fat diet," Journal of Clinical Biochemistry and Nutrition, vol. 46, no. 3, pp. 212-223, 2010.

[34] A. Ferramosca, A. Conte, and V. Zara, "Krill oil ameliorates mitochondrial dysfunctions in rats treated with high-fat diet," BioMed Research International, vol. 2015, Article ID 645984, 2015. 
[35] R. Gallego-Durán, P. Cerro-Salido, E. Gomez-Gonzalez et al., "Imaging biomarkers for steatohepatitis and fibrosis detection in non-alcoholic fatty liver disease," Scientific Reports, vol. 6, Article ID 31421, 2016.

[36] J. K. C. Lau, X. Zhang, and J. Yu, "Animal models of nonalcoholic fatty liver disease: current perspectives and recent advances," The Journal of Pathology, vol. 241, no. 1, pp. 36-44, 2017.

[37] R. K. Schindhelm, M. Diamant, J. M. Dekker, M. E. Tushuizen, T. Teerlink, and R. J. Heine, "Alanine aminotransferase as a marker of non-alcoholic fatty liver disease in relation to type 2 diabetes mellitus and cardiovascular disease," Diabetes/Metabolism Research and Reviews, vol. 22, no. 6, pp. 437443, 2006.

[38] J. M. Clark, F. L. Brancati, and A. M. Diehl, "The prevalence and etiology of elevated aminotransferase levels in the United States," American Journal of Gastroenterology, vol. 98, no. 5, pp. 960-967, 2003.

[39] C.-H. Chen, M.-H. Huang, J.-C. Yang et al., "Prevalence and etiology of elevated serum alanine aminotransferase level in an adult population in Taiwan," Journal of Gastroenterology and Hepatology, vol. 22, no. 9, pp. 1482-1489, 2007.

[40] I. Metón, M. Egea, F. Fernández, M. C. Eraso, and I. V. Baanante, "The N-terminal sequence directs import of mitochondrial alanine aminotransferase into mitochondria," FEBS Letters, vol. 566, no. 1-3, pp. 251-254, 2004.

[41] G. Musso, R. Gambino, and M. Cassader, "Cholesterol metabolism and the pathogenesis of non-alcoholic steatohepatitis," Progress in Lipid Research, vol. 52, no. 1, pp. 175-191, 2013.

[42] M. M. Jacome-Sosa and E. J. Parks, "Fatty acid sources and their fluxes as they contribute to plasma triglyceride concentrations and fatty liver in humans," Current Opinion in Lipidology, vol. 25, no. 3, pp. 213-220, 2014.

[43] K. Yamaguchi, L. Yang, S. McCall et al., "Inhibiting triglyceride synthesis improves hepatic steatosis but exacerbates liver damage and fibrosis in obese mice with nonalcoholic steatohepatitis," Hepatology, vol. 45, no. 6, pp. 1366-1374, 2007.

[44] S. K. Koliwad, R. S. Streeper, M. Monetti et al., "DGAT1dependent triacylglycerol storage by macrophages protects mice from diet-induced insulin resistance and inflammation," The Journal of Clinical Investigation, vol. 120, no. 3, pp. 756-767, 2010.

[45] E. Buzzetti, M. Pinzani, and E. A. Tsochatzis, "The multiplehit pathogenesis of non-alcoholic fatty liver disease (NAFLD)," Metabolism - Clinical and Experimental, 2016.

[46] H. Tilg and A. M. Diehl, "Cytokines in alcoholic and nonalcoholic steatohepatitis," The New England Journal of Medicine, vol. 343, no. 20, pp. 1467-1476, 2000.

[47] J. Crespo, A. Cayon, P. Fernandez-Gil et al., "Gene expression of tumor necrosis factor alpha and TNF-receptors, p55 and p75, in nonalcoholic steatohepatitis patients. Hepatology," FernándezEscalante JC and Pons-Romero F. Gene expression of tumor necrosis factor alpha and TNF-receptors, vol. 34, pp. 1158-1163, 2001.

[48] Y. Wei, R. S. Rector, J. P. Thyfault, and J. A. Ibdah, "Nonalcoholic fatty liver disease and mitochondrial dysfunction," World Journal of Gastroenterology, vol. 14, no. 2, pp. 193-199, 2008.

[49] S. Sanjabi, L. A. Zenewicz, M. Kamanaka, and R. A. Flavell, "Anti-inflammatory and pro-inflammatory roles of TGF- $\beta$, IL10 , and IL-22 in immunity and autoimmunity," Current Opinion in Pharmacology, vol. 9, no. 4, pp. 447-453, 2009.
[50] M. M. Shull, I. Ormsby, A. B. Kier et al., "Targeted disruption of the mouse transforming growth factor- $\beta 1$ gene results in multifocal inflammatory disease," Nature, vol. 359, no. 6397, pp. 693-699, 1992.

[51] A. Mishra, A. Chaudhary, and S. Sethi, "Oxidized omega-3 fatty acids inhibit NF- $\kappa \mathrm{B}$ activation via a PPAR $\alpha$-dependent pathway," Arteriosclerosis, Thrombosis, and Vascular Biology, vol. 24, no. 9, pp. 1621-1627, 2004.

[52] R. Stienstra, S. Mandard, D. Patsouris, C. Maass, S. Kersten, and M. Müller, "Peroxisome proliferator-activated receptor $\alpha$ protects against obesity-induced hepatic inflammation," Endocrinology, vol. 148, no. 6, pp. 2753-2763, 2007.

[53] E. Bettelli, Y. Carrier, W. Gao et al., "Reciprocal developmental pathways for the generation of pathogenic effector TH17 and regulatory T cells," Nature, vol. 441, no. 7090, pp. 235-238, 2006.

[54] D. Pessayre and B. Fromenty, "NASH: a mitochondrial disease," Journal of Hepatology, vol. 42, no. 6, pp. 928-940, 2005.

[55] D. C. Andrade, S. N. Carvalho, D. Pinheiro et al., "Bone marrow mononuclear cell transplantation improves mitochondrial bioenergetics in the liver of cholestatic rats," Experimental Cell Research, vol. 336, no. 1, pp. 15-22, 2015.

[56] J. D. Browning and J. D. Horton, "Molecular mediators of hepatic steatosis and liver injury," The Journal of Clinical Investigation, vol. 114, no. 2, pp. 147-152, 2004.

[57] E. M. Ghamry and N. A. El-Boghdady, "Oxidative stress in nonalcoholic fatty liver disease," Al-Azhar Assiut Medical Journal, vol. 13, pp. 26-35, 2015.

[58] K. Lahbib and S. Touil, "Effect of 3-keto-1,5-bisphosphonates on obese-liver's rats," Biomedicine \& Pharmacotherapy, vol. 83, pp. 186-193, 2016.

[59] S. N. Al-Shaaibi, M. I. Waly, L. Al-Subhi, M. H. Tageldin, N. M. Al-Balushi, and M. S. Rahman, "Ameliorative effects of pomegranate peel extract against dietary-induced nonalcoholic fatty liver in rats," Preventive Nutrition and Food Science, vol. 21, no. 1, pp. 14-23, 2016.

[60] C. Lambert, J. Cubedo, T. Padró et al., "Phytosterols and omega 3 supplementation exert novel regulatory effects on metabolic and inflammatory pathways: A proteomic study," Nutrients, vol. 9, no. 6, article no. 599, 2017.

[61] M. Veselinovic, D. Vasiljevic, V. Vucic et al., "Clinical benefits of n-3 PUFA and $\gamma$-linolenic acid in patients with rheumatoid arthritis," Nutrients, vol. 9, no. 4, article no. 325, 2017.

[62] A. J. Sanyal, M. F. Abdelmalek, A. Suzuki, O. W. Cummings, and M. Chojkier, "No significant effects of ethyl-eicosapentanoic acid on histologic features of nonalcoholic steatohepatitis in a phase 2 trial," Gastroenterology, vol. 147, no. 2, pp. 377-384.el, 2014.

[63] C. K. Argo, J. T. Patrie, C. Lackner et al., "Effects of n-3 fish oil on metabolic and histological parameters in NASH: a double-blind, randomized, placebo-controlled trial," Journal of Hepatology, vol. 62, no. 1, pp. 190-197, 2014.

[64] A. Ferramosca, A. Conte, L. Burri et al., "A krill oil supplemented diet suppresses hepatic steatosis in high-fat fed rats," PLoS ONE, vol. 7, no. 6, Article ID e38797, 2012.

[65] J.-M. Shih, Y.-M. Shih, M.-H. Pai, Y.-C. Hou, C.-L. Yeh, and S.L. Yeh, "Fish oil-based fat emulsion reduces acute kidney injury and inflammatory response in antibiotic-treated polymicrobial septic mice," Nutrients, vol. 8, no. 3, article no. 165, 2016. 


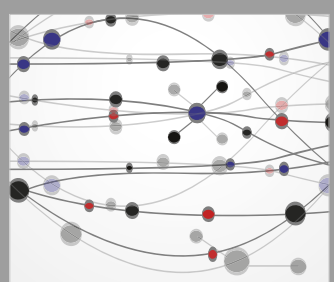

The Scientific World Journal
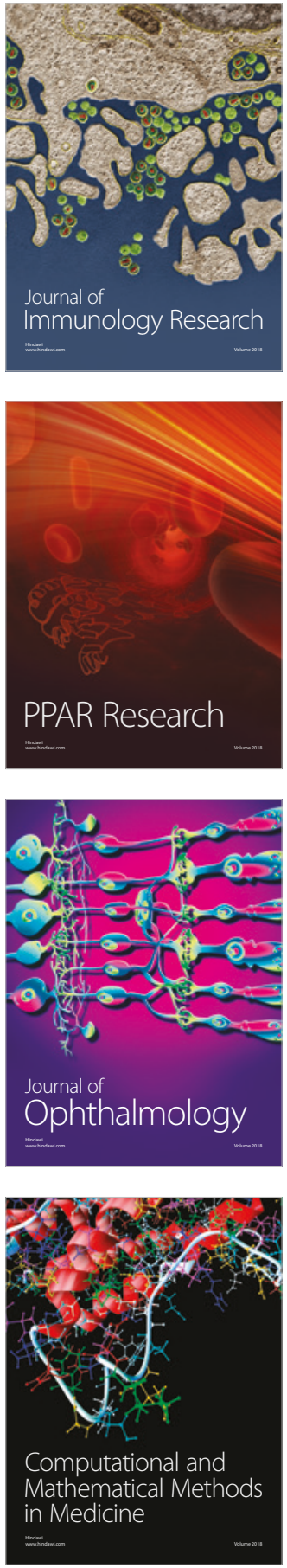

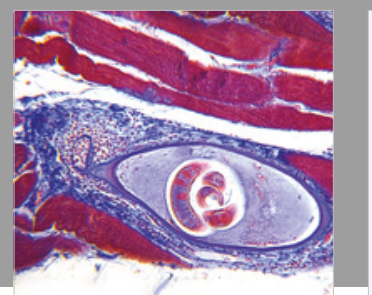

Gastroenterology Research and Practice

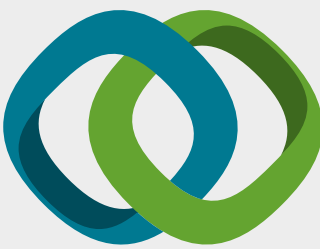

\section{Hindawi}

Submit your manuscripts at

www.hindawi.com
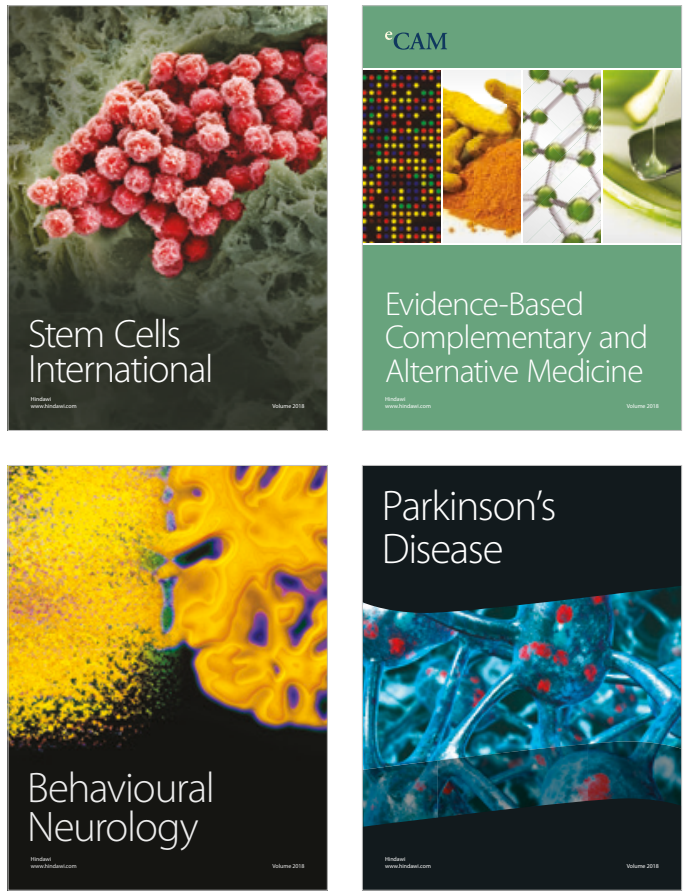

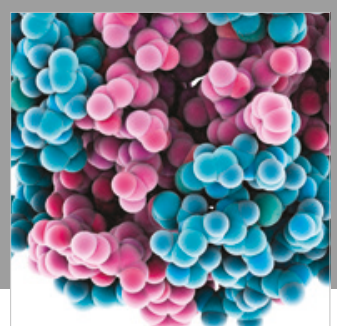

ournal of

Diabetes Research

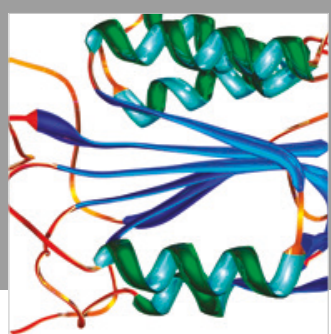

Disease Markers
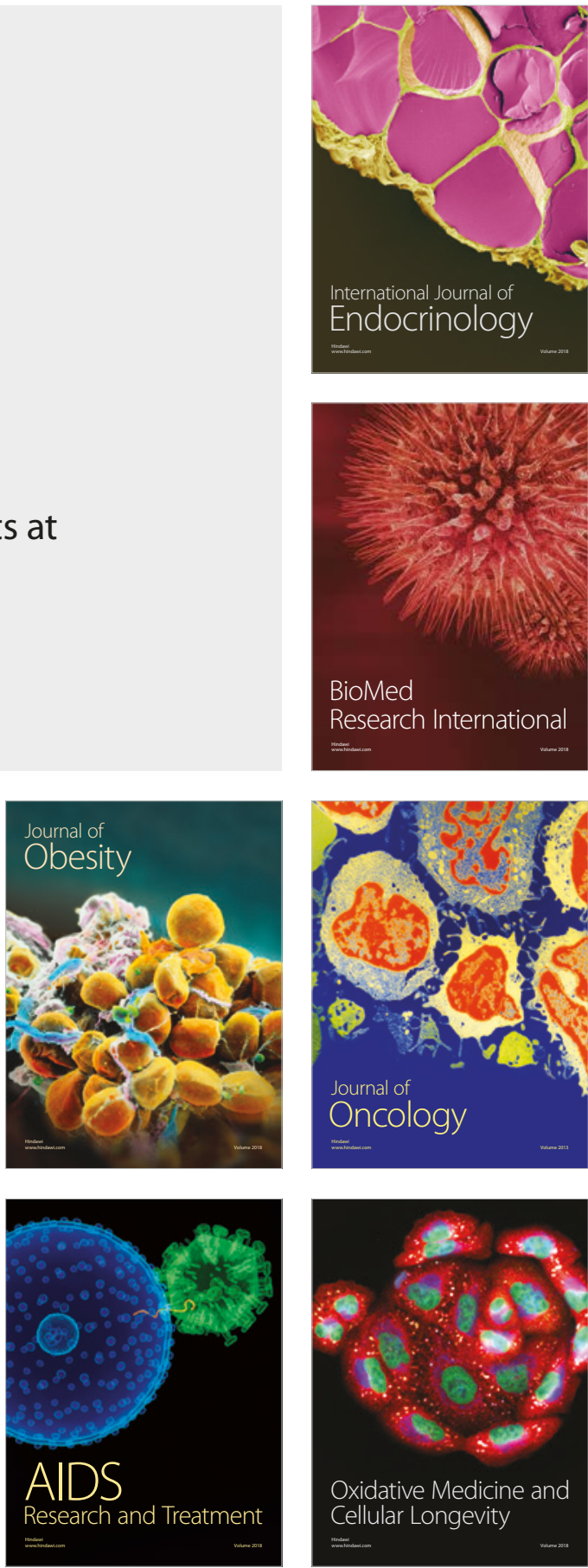